\title{
Linear Boundary Value Problems for Generalized Differential Equations
}

\author{
Milan Tvrdý * \\ Dedicated to the memory of Zdzistaw Wyderka \\ Mathematical Institute \\ Academy of Sciences of the Czech Republic \\ 11567 PRAHA 1, Žitná 25, Czech Republic \\ (e-mail: tvrdy@math.cas.cz)
}

Abstract. The paper deals with boundary value problems of the form

$$
\begin{gathered}
x(t)-x(0)-\int_{0}^{t} \mathrm{~d}[A(s)] x(s)=f(t)-f(0), \quad t \in[0,1], \\
M x(0)+\int_{0}^{1} K(\tau) \mathrm{d}[x(\tau)]=r .
\end{gathered}
$$

Their solutions are functions regulated on $[0,1]$ and regular on $(0,1)$ (i.e. $2 x(t)=x(t-)+x(t+)$ for all $t \in(0,1)$ ). We assume that $A$ and $K$ have bounded variations on $[0,1], f$ is regulated on $[0,1]$ and all of them are regular on $(0,1)$. We derive conditions for the existence and uniqueness of solutions to the given problem. Furthermore, the relationship between the dimensions of the spaces of solutions of the corresponding homogeneous problem and of its adjoint is established. Special attention is paid to the case when the additional condition $(0.2)$ reduces to the periodic boundary condition $x(0)=x(1)$. It is known (cf. [13]) that in the case that $A$ and $f$ are continuous from the right at $t=0$ and from the left at $t=1$, the equation (0.1) reduces to the distributional differential equation

$$
x^{\prime}-A^{\prime} x=f^{\prime} .
$$

Related results concerning the case of solutions left-continuous on $(0,1)$ were obtained in [18] and similar questions for periodic problems and for linear differential equations with distributional coefficients of the form (0.3) were recently treated by Z. Wyderka [21], cf. also [2], [3] or [10].

AMS Subject Classification. 45A05, 47G10, 26A39, 26A42.

Keywords. Generalized linear differential equation, boundary value problem, distribution, periodic solution, equations with impulses, Perron-Stieltjes integral, Henstock-Kurzweil integral.

\footnotetext{
${ }^{*}$ Supported by the grant No. 201/97/0218 of the Grant Agency of the Czech Republic
} 


\section{Preliminaries}

Throughout the paper $\mathbb{R}^{n \times m}$ denotes the space of real $n \times m$-matrices, $\mathbb{R}^{n}=\mathbb{R}^{n \times 1}$, $R^{1}=R$. Given an $n \times m$-matrix $A \in \mathbb{R}^{n \times m}$, its elements are denoted by $a_{i, j}$, $\operatorname{det}(A)$ and $\operatorname{rank}(A)$ denote respectively its determinant and its rank, $A^{\mathrm{T}}$ stands for its transposition and $|A|=\max _{i=1, \ldots, n} \sum_{j=1}^{m}\left|a_{i, j}\right|$ is its norm, (In particular, $y^{\mathrm{T}}=\left(y_{1}, y_{2}, \ldots, y_{n}\right)$ for $y \in \mathbb{R}^{n}$.) The symbols I and 0 stand respectively for the identity and the zero matrix of the proper type.

For given $n \times n$-matrices $C_{j}, j=1,2, \ldots, p$, the symbol $\prod_{j=1}^{p} C_{j}$ is defined by

$$
\prod_{j=1}^{p} C_{j}=C_{1} C_{2} \ldots C_{p}, \quad \text { while } \quad \prod_{j=0}^{p-1} C_{p-j}=C_{p} C_{p-1} \ldots C_{1}
$$

As usual, by $[0,1]$ and $(0,1)$ we denote the corresponding closed and open intervals, respectively. Furthermore, $[0,1)$ and $(0,1]$ are the corresponding halfopen intervals.

Any function $F:[0,1] \mapsto \mathbb{R}^{n \times m}$ which possesses finite limits

$$
F(t+)=\lim _{\tau \rightarrow t+} F(\tau) \quad \text { and } \quad F(s-)=\lim _{\tau \rightarrow s-} F(\tau)
$$

for all $t \in[0,1)$ and $s \in(0,1]$ is said to be regulated on $[0,1]$. The linear space of $n \times m$-matrix valued functions regulated on $[0,1]$ is denoted by $\mathbb{G}^{n \times m}$, while $\mathbb{G}_{\text {reg }}^{n \times m}$ stands for the space of functions $F$ from $\mathbb{G}^{n \times m}$ which are regular on $(0,1)$, i.e. which satisfy the relations

$$
F(t)=\frac{F(t-)+F(t+)}{2}, \quad t \in(0,1)
$$

Instead of $\mathbb{G}^{n \times 1}$ we write $\mathbb{G}^{n}$. Analogously, $\mathbb{G}_{\text {reg }}^{n \times 1}=\mathbb{G}_{\text {reg }}^{n}$. For $x \in \mathbb{G}^{n}$ we put

$$
\|x\|=\sup _{t \in[0,1]}|x(t)| \text {. }
$$

It is well known that both $\mathbb{G}^{n}$ and $\mathbb{G}_{\text {reg }}^{n}$ are Banach spaces with respect to this norm (cf. [7, Theorem 3.6]). Given $F \in \mathbb{G}^{n \times m}$, we put $F(0-)=F(0)$ and $F(1+)=F(1)$ and, for any $t \in[0,1]$, we define

$$
\Delta^{+} F(t)=F(t+)-F(t), \quad \Delta^{-} F(t)=F(t)-F(t-)
$$

and

$$
\Delta F(t)=F(t+)-F(t-) .
$$

As usual, the space of $n \times m$-matrix valued functions continuous on $[0,1]$ is denoted by $\mathbb{C}^{n \times m}$. 
For a function $F:[0,1] \mapsto \mathbb{R}^{n \times m}$ and a subdivision $D=\left\{0=\alpha_{0}<\alpha_{1}<\right.$ $\left.\cdots<\alpha_{k}=1\right\}$ of the interval $[0,1]$, we put

$$
v(F, D)=\sum_{j=1}^{k}\left|F\left(\alpha_{j}\right)-F\left(\alpha_{j-1}\right)\right| \quad \text { and } \quad \operatorname{var}_{0}^{1} F=\sup _{D} v(F, D),
$$

where the supremum is taken over all subdivisions $D$ of $[0,1]$. The space of all functions $F:[0,1] \mapsto \mathbb{R}^{n \times m}$ such that $\operatorname{var}_{0}^{1} F<\infty$ is denoted by $\mathbb{B V}^{n \times m}$. It is well known that $\mathbb{B} \mathbb{V}^{n \times m}$ equipped with the norm

$$
F \in \mathbb{B V}^{n \times m} \mapsto\|F\|_{\mathbb{B V}}=|F(0)|+\operatorname{var}_{0}^{1} F
$$

is a Banach space. Obviously, $F \in \mathbb{B V}^{n \times m}$ if and only if all its components $a_{i, j}$ have a bounded variation on $[0,1]$. The space of all functions $F \in \mathbb{B V}^{n \times m}$ which are regular on $[0,1]$ (i.e. satisfy the relation (1.1)) is denoted by $\mathbb{B V}_{\text {reg }}^{n \times m}$. Instead of $\mathbb{B V}^{n \times 1}$ or $\mathbb{B V}_{\text {reg }}^{n \times 1}$ we write $\mathbb{B V}^{n}$ or $\mathbb{B V}_{\text {reg }}^{n}$, respectively.

For more details concerning regulated functions or functions of bounded variation see [1], [7], [4] or [6], respectively.

For given linear spaces $\mathbb{X}$ and $\mathbb{Y}$, the symbol $\mathcal{L}(\mathbb{X}, \mathbb{Y})$ denotes the linear space of linear bounded mappings of $\mathbb{X}$ into $\mathbb{Y}$. If $\mathrm{L} \in \mathcal{L}(\mathbb{X}, \mathbb{Y})$ then $\mathcal{R}(\mathrm{L}), \mathcal{N}(\mathrm{L})$ and $\mathrm{L}^{*}$ denote its range, null space and adjoint operator, respectively. For a given linear bounded functional $\xi \in \mathbb{X}^{*}$, its value on $x \in \mathbb{X}$ is denoted by $\langle x, \xi\rangle_{\mathbb{X}}$.

The integrals which occur in this paper are the Perron-Stieltjes ones. In particular, we make use of the equivalent definition of these integrals due to J. Kurzweil (cf. e.g. [8], [9], [15] and [16]). Let us recall here that if $A \in \mathbb{G}^{n \times n}$, $x \in \mathbb{G}^{n}$ and at least one of them has a bounded variation on $[0,1]$ then the integral

$$
\int_{0}^{t} \mathrm{~d}[A(\tau)] x(\tau)
$$

exists for any $t \in[0,1]$ and the function

$$
h: t \in[0,1] \mapsto \int_{0}^{t} \mathrm{~d}[A(\tau)] x(\tau) \in \mathbb{R}^{n}
$$

is regulated on $[0,1]$ (cf. [17, Theorem 2.8]). Moreover, if $A \in \mathbb{B V}^{n \times n}$ then $h \in \mathbb{B V}^{n \times n}$ and if $A \in \mathbb{B V}_{\text {reg }}^{n \times n}$ then $h \in \mathbb{B V}_{\text {reg }}^{n}$, as well. Finally, let us recall that by [19, Theorem 2.7] the left hand side of the additional condition

$$
M x(0)+\int_{0}^{1} K(\tau) \mathrm{d}[x(\tau)]=r
$$


represents the general form of a linear bounded mapping of the space $\mathbb{G}_{\text {reg }}^{n}$ into $\mathbb{R}^{m}$. Some further details concerning the integration with respect to regulated functions may be found in [17].

Distributions are considered in the sense of L. Schwartz, i.e. as linear continuous ( $n$-vector valued) functionals on the topological vector space $\mathcal{D}^{n}$ of functions $\varphi: \mathbb{R} \mapsto \mathbb{R}^{n}$ possessing for any $j \in \mathbb{N} \cup\{0\}$ a derivative $\varphi^{(j)}$ of the order $j$ which is continuous on $\mathbb{R}$ and such that $\varphi^{(j)}(t)=0$ for any $t \in \mathbb{R} \backslash(0,1)$. The space $\mathcal{D}^{n}$ is endowed with the topology in which the sequence $\varphi_{k} \in \mathcal{D}^{n}$ tends to $\varphi_{0} \in \mathcal{D}^{n}$ in $\mathcal{D}^{n}$ if and only if

$$
\lim _{k \rightarrow \infty}\left\|\varphi_{k}^{(j)}-\varphi_{0}^{(j)}\right\|=0
$$

for all non negative integers $j$. The space of distributions on $[0,1]$ (i.e. the dual space to $\left.\mathcal{D}^{n}\right)$ is denoted by $\mathcal{D}^{n *}$. The zero distribution $0 \in \mathcal{D}^{n *}$ on $[0,1]$ is identified with an arbitrary measurable function vanishing a.e. on $[0,1]$. Obviously, if $f \in \mathbb{G}$ then $f=0 \in \mathcal{D}^{n *}$ only if $f(t-)=f(s+)=0$ for all $t \in(0,1]$ and all $s \in[0,1)$. Consequently, if $f \in \mathbb{G}_{\text {reg }}^{n}$ and $f(0+)=f(0)$ and $f(1-)=f(1)$, then $f=0 \in \mathcal{D}^{n *}$ if and only if $f(t)=0$ for all $t \in[0,1]$. This means that for a given function $g$ Lebesgue integrable on $[0,1]$ there may exist at most one function $f \in \mathbb{G}_{\text {reg }}^{n}$ such that $f(0+)=f(0), f(1-)=f(1)$ and $f(t)=g(t)$ a.e. on $[0,1]$. For a given $f \in \mathcal{D}^{n *}, f^{\prime}$ denotes its distributional derivative, i.e.

$$
f^{\prime}: \varphi \in \mathcal{D}^{n} \mapsto\left\langle f^{\prime}, \varphi\right\rangle_{\mathcal{D}^{n}}=-\left\langle f, \varphi^{\prime}\right\rangle_{\mathcal{D}^{n}}
$$

For more details concerning distributions see e.g. [5] or [14].

Similarly as in [11] we define for given $x \in \mathbb{G}_{\text {reg }}^{n}$ and $A \in \mathbb{B V}_{\text {reg }}^{n \times n}$

$$
A^{\prime} x: \varphi \in \mathcal{D}^{n} \mapsto\left\langle A^{\prime} x, \varphi\right\rangle_{\mathcal{D}^{n}}=\int_{0}^{1} \varphi^{\mathrm{T}}(t) \mathrm{d}\left[\int_{0}^{t} \mathrm{~d}[A(\tau)] x(\tau)\right]
$$

and

$$
A x^{\prime}: \varphi \in \mathcal{D}^{n} \mapsto\left\langle A x^{\prime}, \varphi\right\rangle_{\mathcal{D}^{n}}=\int_{0}^{1} \varphi^{\mathrm{T}}(t) \mathrm{d}\left[\int_{0}^{t} A(t) \mathrm{d}[x(t)]\right]
$$

It follows (cf. [13]) that the relations

$$
A^{\prime} x=\left(\int_{0}^{t} \mathrm{~d}[A(\tau)] x(\tau)\right)^{\prime} \quad \text { and } \quad A x^{\prime}=\left(\int_{0}^{t} A(\tau) \mathrm{d}[x(\tau)]\right)^{\prime}
$$

are true. Making use of the integration-by-parts formula (cf. [17, Proposition $1.2])$ it is easy to verify that for any couple of functions $x \in \mathbb{G}_{\text {reg }}^{n}, A \in \mathbb{B V}_{\text {reg }}^{n \times n}$ the relation $(A x)^{\prime}=A x^{\prime}+A^{\prime} x$ is true (cf. [13]). 


\section{2 . General boundary value problem}

Throughout the paper we assume

2.1. Assumptions. $0 \leq m \leq 2 n, A \in \mathbb{B V}_{\text {reg }}^{n \times n}, f \in \mathbb{G}_{\text {reg }}^{n}, M \in \mathbb{R}^{m \times n}, K \in$ $\mathbb{B V}^{m \times n}, r \in \mathbb{R}^{m}$ and

$$
\operatorname{det}\left(\mathrm{I}+\Delta^{+} A(0)\right) \operatorname{det}\left(\mathrm{I}-\left[\Delta^{-} A(t)\right]^{2}\right) \operatorname{det}\left(\mathrm{I}-\Delta^{-} A(1)\right) \neq 0
$$

for all $t \in(0,1)$.

We will consider the boundary value problem (0.1), (0.2). An $n$-vector valued function $x:[0,1] \mapsto \mathbb{R}^{n}$ is said to be its solution if it belongs to $\mathbb{G}_{\text {reg }}^{n}$ and satisfies $(0.1)$ and $(0.2)$.

It is known (cf. [13, Proposition 2.3]) that if

$$
A(0+)=A(0), f(0+)=f(0), A(1-)=A(1) \text { and } f(1-)=f(1),
$$

then $x \in \mathbb{G}_{\text {reg }}^{n}$ is a solution to $(0.1)$ on $[0,1]$ if and only if the relation

$$
\int_{0}^{1} \varphi^{\mathrm{T}}(t) \mathrm{d}\left[x(t)-\int_{0}^{t} \mathrm{~d}[A(\tau)] x(\tau)-f(t)\right]=0
$$

holds for all $\varphi \in \mathcal{D}^{n}$, i.e. if and only if $x^{\prime}-A^{\prime} x-f^{\prime}$ is the zero distribution. In other words, if (2.1) is true, then the equation (0.1) is equivalent to the distributional differential equation (0.3).

It is also known (cf. e.g. [16, Section III.2] or [15, Theorems 6.15 and 6.17]) that under our assumptions there exists $U:[0,1] \times[0,1] \mapsto \mathbb{R}^{n \times n}$ such that $x:[0,1] \mapsto \mathbb{R}^{n}$ is a solution to $(0.1)$ on $[0,1]$ if and only if

$$
\begin{aligned}
x(t)=U( & (t, 0) x(0)+f(t)-f(0) \\
& -\int_{0}^{t} \mathrm{~d}_{\tau}[U(t, \tau)](f(\tau)-f(0)) \text { on }[0,1] .
\end{aligned}
$$

The function $U$ is uniquely determined by the relations

$$
U(t, s)=\mathrm{I}+\int_{s}^{t} \mathrm{~d}[A(\tau)] U(\tau, s) \quad \text { for all } t, s \in[0,1] .
$$

Furthermore, it satisfies the relations

$$
U(t, \tau) U(\tau, s)=U(t, s) \text { and } \operatorname{det}(U(t, s)) \neq 0 \text { for all } t, s, \tau \in[0,1] .
$$

Inserting (2.2) into (0.2) we get the following assertion. 
2.2. Proposition. Under the assumptions 2.1 the problem $(0.1),(0.2)$ possesses a unique solution for any $f \in \mathbb{G}_{\text {reg }}^{n}$ and any $r \in \mathbb{R}^{m}$ if and only if

$$
m=n \quad \text { and } \quad \operatorname{dim} \mathcal{N}(\mathrm{L})=0,
$$

or equivalently if and only if

$$
m=n \quad \text { and } \quad \operatorname{det}\left(M+\int_{0}^{1} K(\tau) \mathrm{d}_{\tau}[U(\tau, 0)]\right) \neq 0
$$

Proof. The problem (0.1), (0.2) obviously possesses a solution if and only if there is $c \in \mathbb{R}^{n}$ such that

$$
D c=b,
$$

where

$$
D=M+\int_{0}^{1} K(\tau) \mathrm{d}_{\tau}[U(\tau, 0)]
$$

and

$$
\left.b=r-\int_{0}^{1} K(\tau) d[f(\tau)]+\int_{0}^{1} K(\tau) \mathrm{d}_{\tau}\left[\int_{0}^{\tau} \mathrm{d}_{s}[U(\tau, s)] f(s)-f(0)\right)\right],
$$

and this solution is then given by (2.2), where we put $x(0)=c$. Consequently, the problem $(0.1),(0.2)$ possesses for any $(f, r) \in \mathbb{G}_{\text {reg }}^{n} \times \mathbb{R}^{m}$ a unique solution if and only if for any $b \in \mathbb{R}^{m}$ the equation (2.6) possesses a unique solution $c \in \mathbb{R}^{n}$. Obviously, this is possible if and only if $m=n$ and the homogeneous equation $D c=0$ possesses only the trivial solution $c=0$, i.e. if and only if $(2.5)$ is true or equivalently if and only if (2.4) is true.

2.3. Definition. For given $x \in \mathbb{G}_{\text {reg }}^{n}$ and $t \in[0,1]$, we define

$$
(\mathrm{L} x)(t)=\left(\begin{array}{c}
\left.x(t)-x(0)-\int_{0}^{t} \mathrm{~d}[A(\tau)] \tau\right) \\
M x(0)+\int_{0}^{1} K(\tau) d[x(\tau)]
\end{array}\right) .
$$

Using Definition 2.3 we can rewrite the problem $(0.1),(0.2)$ as the operator equation

$$
\mathrm{L} x=\left(\begin{array}{c}
f(t)-f(0) \\
r
\end{array}\right)
$$


Furthermore, $\mathrm{L} \in \mathcal{L}\left(\mathbb{G}^{n}, \mathbb{G}^{n} \times \mathbb{R}^{m}\right)$ (cf. [17, Proposition 2.16 and Theorem 2.8]). As we noticed in Section 1 the function $h$ given by

$$
h: t \in[0,1] \mapsto \int_{0}^{t} \mathrm{~d}[A(\tau)] x(\tau) \in \mathbb{R}^{n}
$$

belongs to $\mathbb{G}_{\text {reg }}^{n}$ for any $x \in \mathbb{G}^{n}$. Consequently, $\mathrm{L} \in \mathcal{L}\left(\mathbb{G}_{\text {reg }}^{n}, \mathbb{G}_{\text {reg }}^{n} \times \mathbb{R}^{m}\right)$. Moreover, analogously as it was done for the case of solutions left-continuous on $(0,1]$ in the proof of [18, Proposition 2.6] we could utilize the formula (2.2) to show the following assertion.

2.4. Theorem. Let us assume 2.1 and let the operator $\mathrm{L}$ be given by (2.7). Then the range $\mathcal{R}(\mathrm{L})$ of the operator $\mathrm{L}$ is closed in $\mathbb{G}_{\text {reg }}^{n} \times \mathbb{R}^{m}$.

Furthermore, in virtue of [19, Theorem 2.7], $\mathbb{B V} \mathbb{V}^{n} \times \mathbb{R}^{n} \times \mathbb{R}^{m}$ is the dual space to $\mathbb{G}_{\text {reg }}^{n} \times \mathbb{R}^{m}$, while for given $y \in \mathbb{B V}^{n}, \gamma \in \mathbb{R}^{n}$ and $\delta \in \mathbb{R}^{m}$, the corresponding linear bounded functional is given by

$$
\begin{aligned}
(g, r) \in \mathbb{G}_{\mathrm{reg}}^{n} & \times \mathbb{R}^{m} \mapsto \\
& \langle(g, r),(y, \gamma, \delta)\rangle_{\mathbb{G}_{\mathrm{reg}}^{n} \times \mathbb{R}^{m}}:=\gamma^{\mathrm{T}} g(0)+\int_{0}^{1} y^{\mathrm{T}}(\tau) \mathrm{d}[g(\tau)]+\delta^{\mathrm{T}} r .
\end{aligned}
$$

Let $x \in \mathbb{G}_{\text {reg }}^{n}, y \in \mathbb{B V}^{n}, \gamma \in \mathbb{R}^{n}$ and $\delta \in \mathbb{R}^{m}$ be given. Then in virtue of (2.8) and of the Substitution Theorem (cf. [17, Theorem 2.19]) we have

$$
\begin{aligned}
\langle\mathrm{L} x,(y, \gamma, \delta)\rangle_{\mathbb{G}_{\mathrm{reg}}^{n} \times \mathbb{R}^{m}}=\int_{0}^{1}\left(y^{\mathrm{T}}(t)+\delta^{\mathrm{T}} K(t)\right) \mathrm{d}[x(t)] \\
+\delta^{\mathrm{T}} M x(0)+\int_{0}^{1} \mathrm{~d}\left[\int_{t}^{1} y^{\mathrm{T}}(\tau) \mathrm{d}[A(\tau)]\right] x(t) .
\end{aligned}
$$

Furthermore, integrating by parts (cf. [17, Theorem 2.15]) we obtain

$$
\begin{aligned}
\int_{0}^{1} \mathrm{~d}[ & \left.\int_{t}^{1} y^{\mathrm{T}}(\tau) \mathrm{d}[A(\tau)]\right] x(t) \\
= & -\left(\int_{0}^{1} y^{\mathrm{T}}(\tau) \mathrm{d}[A(\tau)]\right) x(0)-\int_{0}^{1}\left(\int_{t}^{1} y^{\mathrm{T}}(\tau) \mathrm{d}[A(\tau)]\right) \mathrm{d}[x(t)] \\
& +y^{\mathrm{T}}(0) \Delta^{+} A(0) \Delta^{+} x(0)-y^{\mathrm{T}}(1) \Delta^{-} A(1) \Delta^{-} x(1) .
\end{aligned}
$$

Define

$$
\widetilde{A}(t)=\left\{\begin{array}{cl}
A(0+) & \text { if } t=0 \\
A(t) & \text { if } t \in(0,1) \\
A(1-) & \text { if } t=1
\end{array}\right.
$$


Then making use of [17, Corollary 2.14] for any $t \in[0,1]$ we get

$$
\int_{t}^{1} y^{\mathrm{T}}(\tau) \mathrm{d}[(\widetilde{A}(\tau)-A(\tau))]=-y^{\mathrm{T}}(1) \Delta^{-} A(1)+z^{\mathrm{T}}(t)
$$

where

$$
z^{\mathrm{T}}(t)=\left\{\begin{array}{cl}
-y^{\mathrm{T}}(0) \Delta^{+} A(0) & \text { if } t=0, \\
0 & \text { if } t \in(0,1), \\
y^{\mathrm{T}}(1) \Delta^{-} A(1) & \text { if } t=1 .
\end{array}\right.
$$

On the other hand, according to [17, Proposition 2.12] we have

$$
\int_{0}^{1} z^{\mathrm{T}}(t) \mathrm{d}[x(t)]=y^{\mathrm{T}}(1) \Delta^{-} A(1) \Delta^{-} x(1)-y^{\mathrm{T}}(0) \Delta^{+} A(0) \Delta^{+} x(0) .
$$

Thus, with respect to (2.12), we have

$$
\begin{aligned}
y^{\mathrm{T}}(1) & \Delta^{-} A(1) \Delta^{-} x(1)-y^{\mathrm{T}}(0) \Delta^{+} A(0) \Delta^{+} x(0) \\
& =-\int_{0}^{1}\left(\int_{t}^{1} y^{\mathrm{T}}(\tau) \mathrm{d}[\widetilde{A}(\tau)-A(\tau)]+y^{\mathrm{T}}(1) \Delta^{-} A(1)\right) \mathrm{d}[x(t)]
\end{aligned}
$$

and the relation $(2.10)$ reduces to

$$
\begin{gathered}
\int_{0}^{1} \mathrm{~d}\left[\int_{t}^{1} y^{\mathrm{T}}(\tau) \mathrm{d}[A(\tau)]\right] x(t)=-\left(\int_{0}^{1} y^{\mathrm{T}}(\tau) \mathrm{d}[A(\tau)]\right) x(0) \\
-\int_{0}^{1}\left(\int_{t}^{1} y^{\mathrm{T}}(\tau) \mathrm{d}[\tilde{A}(\tau)]-y^{\mathrm{T}}(1) \Delta^{-} A(1)\right) \mathrm{d}[x(t)] .
\end{gathered}
$$

Thus

$$
\begin{aligned}
\langle\mathrm{L} x & ,(y, \gamma, \delta)\rangle_{\mathbb{G}_{\mathrm{reg}}^{n} \times \mathbb{R}^{m}} \\
= & \int_{0}^{1}\left(y^{\mathrm{T}}(t)+\delta^{\mathrm{T}} K(t)-\int_{t}^{1} y^{\mathrm{T}}(\tau) \mathrm{d}[\widetilde{A}(\tau)]-y^{\mathrm{T}}(1) \Delta^{-} A(1)\right) \mathrm{d}[x(t)] \\
& +\left(\delta^{\mathrm{T}} M-\int_{0}^{1} y^{\mathrm{T}}(t) \mathrm{d}[A(t)]\right) x(0) .
\end{aligned}
$$

With respect to the definition of the adjoint operator this completes the proof of the following assertion.

2.5. Theorem. Let us assume 2.1. Then the operator

$$
\begin{aligned}
& \stackrel{*}{\mathrm{~L}}:\left(y^{T}, \gamma^{T}, \delta^{T}\right) \in \mathbb{B V}^{n} \times \mathbb{R}^{n} \times \mathbb{R}^{m} \mapsto \\
&\left(y^{T}(t)+\delta^{T}\right. K(t)-\int_{t}^{1} y^{T}(\tau) \mathrm{d}[\widetilde{A}(\tau)]-y^{T}(1) \Delta^{-} A(1), \\
&\left.\delta^{T} M-\int_{0}^{1} y^{T}(\tau) \mathrm{d}[A(\tau)]\right) \quad \in \mathbb{B V}^{n} \times \mathbb{R}^{n}
\end{aligned}
$$

is the adjoint operator to $\mathrm{L}$. 
2.6. Corollary. Let us assume 2.1 and let $\widetilde{A}$ be given by (2.11). Then the problem (0.1), (0.2) possesses a solution in $\mathbb{G}_{\text {reg }}^{n}$ if and only if

$$
\int_{0}^{1} y^{T}(\tau) \mathrm{d}[f(\tau)]+\delta^{T} r=0
$$

holds for any couple $(y, \delta) \in \mathbb{B V}^{n} \times \mathbb{R}^{m}$ verifying the system

$$
\begin{array}{rlrl}
y^{T}(s)-y^{T}(1)-\int_{s}^{1} y^{T}(\tau) \mathrm{d}[\widetilde{A}(\tau)]-\delta^{T}(K(1)-K(s)) & =0, s \in[0,1], \\
y^{T}(0)\left[\mathrm{I}+\Delta^{+} A(0)\right]+\delta^{T}[K(0)-M] & & =0, \\
y^{T}(1)\left[\mathrm{I}-\Delta^{-} A(1)\right]+\delta^{T} K(1) & =0 .
\end{array}
$$

Proof. For a given $f \in \mathbb{G}_{\text {reg }}^{n}$ and $t \in[0,1]$, let us put $g(t)=f(t)-f(0)$. Then

$$
\left\langle(g, r),(y, \gamma, \delta\rangle_{\mathbb{G}_{\text {reg }}^{n} \times \mathbb{R}^{m}}=\int_{0}^{1} y^{\mathrm{T}}(\tau) \mathrm{d}[f(\tau)]+\delta^{\mathrm{T}} r\right.
$$

holds for all $f \in \mathbb{G}_{\text {reg }}^{n}, r \in \mathbb{R}^{m}, y \in \mathbb{B V}^{n}, \gamma \in \mathbb{R}^{n}$ and $\delta \in \mathbb{R}^{m}$. Thus, by Theorems 2.4 and 2.5 (cf. (2.8) and (2.10), as well) the problem (0.1), (0.2) (or equivalently the operator equation $\mathrm{L} x=(g, r))$ possesses a solution if and only if (2.13) is true for any solution $(y, \gamma, \delta) \in \mathbb{B V}^{n} \times \mathbb{R}^{n} \times \mathbb{R}^{m}$ of the system

$$
\begin{aligned}
y^{\mathrm{T}}(s) & =\int_{s}^{1} y^{\mathrm{T}}(\tau) \mathrm{d}[\widetilde{A}(\tau)]-\delta^{\mathrm{T}} K(s)-y^{\mathrm{T}}(1) \Delta^{-} A(1) \quad \text { on } \quad[0,1], \\
0 & =\int_{0}^{1} y^{\mathrm{T}}(\tau) \mathrm{d}[A(\tau)]-\delta^{\mathrm{T}} M .
\end{aligned}
$$

(Notice that for $\gamma$ we did not obtain any condition and $\gamma$ does not appear in the condition (2.13), as well.)

Now, making use of (2.12), it is easy to verify that the systems (2.14)-(2.16) and (2.17) are equivalent and this completes the proof.

2.7. Corollary. Let us assume 2.1 and let $\widetilde{A}$ be given by (2.11). Then the periodic boundary value problem (0.1),

$$
x(0)=x(1)
$$

possesses a solution in $\mathbb{G}_{r e g}^{n}$ if and only if

$$
\int_{0}^{1} y^{T}(s) f(s) \mathrm{d} s=0
$$


holds for any solution $y \in \mathbb{B V}^{n}$ of the system

$$
\begin{gathered}
y^{T}(s)=y^{T}(1)+\int_{s}^{1} y^{T}(\tau) \mathrm{d}[\widetilde{A}(\tau)], \quad t \in[0,1], \\
y^{T}(0)\left[\mathrm{I}+\Delta^{+} A(0)\right]=y^{T}(1)\left[\mathrm{I}-\Delta^{-} A(1)\right] .
\end{gathered}
$$

Proof. If we put

$$
M=0 \quad \text { and } \quad K(t) \equiv \mathrm{I} \text { on }[0,1]
$$

then the condition (2.18) takes the form (0.2). Inserting (2.21) into (2.14)-(2.16) we obtain (2.19) and (2.20).

2.8. Definition. The system (2.14)-(2.16) is said to be the adjoint problem to (0.1), (0.2) (or to the corresponding homogeneous problem $\mathrm{L} x=0$ ).

2.9. Remark. Obviously, $y \in \mathbb{B V}_{\text {reg }}^{n}$ whenever $y$ is a solution of (2.19). Hence, making use of the definition (1.2) (cf. also (1.4) ) the equation (2.19) can be rewritten as the distributional differential equation $y^{\prime}=-\left(\widetilde{A}^{\mathrm{T}}\right)^{\prime} y$.

2.10. Remark. If in addition to 2.1 we assumed also $\Delta^{+} A(0)=0$ and $\Delta^{-} A(1)=$ 0 , then the adjoint problems (2.14)-(2.16) and (2.19), (2.20) would reduce to the systems

$$
\text { (2.14), } \quad y^{\mathrm{T}}(0)=-\delta^{\mathrm{T}}(K(0)-M), \quad y^{\mathrm{T}}(1)=-\delta^{\mathrm{T}} K(1)
$$

and

$$
y^{\prime}=-\left(\widetilde{A}^{\mathrm{T}}\right)^{\prime} y, \quad y^{\mathrm{T}}(0)=y^{\mathrm{T}}(1)
$$

respectively.

\section{3 . Adjoint problem}

In this section we will consider the adjoint problem (2.14)-(2.16) to the given problem $(0.1),(0.2)$. In addition to 2.1 we will assume also that

$$
\operatorname{det}\left(\mathrm{I}-\Delta^{+} A(0)\right) \operatorname{det}\left(\mathrm{I}+\Delta^{-} A(1)\right) \neq 0 .
$$

Obviously, under assumptions 2.1, for the function $\widetilde{A}$ given by $(2.11)$ we have

$$
\widetilde{A} \in \mathbb{B V}_{\mathrm{reg}}^{n \times n}, \quad \widetilde{A}(0+)=\widetilde{A}(0), \quad \widetilde{A}(1-)=\widetilde{A}(1)
$$

and 


$$
\operatorname{det}\left(\mathrm{I}-\left(\Delta^{-} \widetilde{A}(s)\right)^{2}\right) \neq 0 \text { on }[0,1] .
$$

(Let us notice that according to the conventions introduced in Section 1, we put $\Delta^{-} \widetilde{A}(0)=\Delta^{+} \widetilde{A}(1)=0$.) Hence, for given $\delta \in \mathbb{R}^{m}$ and $\eta \in \mathbb{R}^{n}$, the equation (2.14) possesses a unique solution $y$ on $[0,1]$ such that $y(1)=\eta$ (cf. [16, Section III.4]). This solution is given on $[0,1]$ by

$$
\begin{array}{rl}
y^{\mathrm{T}}(s)=\eta^{\mathrm{T}} & V(1, s)-\delta^{\mathrm{T}}(K(s)-K(1)) \\
& -\delta^{\mathrm{T}} \int_{s}^{1}(K(\tau)-K(1)) \mathrm{d}_{s}[V(\tau, s)], \quad s \in[0,1],
\end{array}
$$

where $V$ is an $n \times n$-matrix valued function uniquely determined on $[0,1] \times[0,1]$ by the relation

$$
V(t, s)=\mathrm{I}+\int_{s}^{t} V(t, \tau) \mathrm{d}[\widetilde{A}(\tau)], \quad t, s \in[0,1] .
$$

Let $s, t \in[0,1]$ such that $0 \leq s \leq t \leq 1$ be given. Inserting (2.3) and (3.3) into the expression

$$
W(t, s):=\int_{s}^{t} \mathrm{~d}_{\tau}[V(t, \tau)] U(\tau, t)+\int_{s}^{t} V(t, \tau) \mathrm{d}_{\tau}[U(\tau, t)]
$$

and making use of the Substitution Theorem (cf. [17, Theorem 2.19]) we get

$$
\begin{aligned}
W(t, s) & =\int_{s}^{t} V(t, \tau) \mathrm{d}[A(\tau)-\widetilde{A}(\tau)] U(\tau, t) \\
& =V(t, s) \Delta^{+}(A(s)-\widetilde{A}(s)) U(s, t)+\Delta^{-}(A(t)-\widetilde{A}(t)) \\
& =\left\{\begin{array}{cc}
-\Delta^{+} A(0) & \text { if } s=0 \\
0 & \text { if } s>0
\end{array}\right\}+\left\{\begin{array}{cc}
0 & \text { if } t<1 \\
\Delta^{-} A(1) & \text { if } t=1
\end{array}\right\} .
\end{aligned}
$$

On the other hand, the integration-by-parts formula when applied to $W(t, s)$ yields

$$
\begin{array}{r}
W(t, s)=\mathrm{I}-V(t, s) U(s, t)+\Delta^{-} \widetilde{A}(t) \Delta^{-} A(t) \\
-V(t, s) \Delta^{+} \widetilde{A}(s) \Delta^{+} A(s) U(s, t),
\end{array}
$$

where the relations

$$
\begin{array}{lll}
U(t+, s)-U(t, s)=\Delta^{+} A(t) U(t, s), & t \in(0,1], s \in[0,1], \\
V(t, s+)-V(t, s)=-V(t, s) \Delta^{+} \widetilde{A}(s), & t \in[0,1], s \in[0,1), \\
U(t, s)-U(t-, s)=\Delta^{-} A(t) U(t, s), & t \in(0,1], s \in[0,1], \\
V(t, s)-V(t, s-)=-V(t, s) \Delta^{-} \widetilde{A}(s) & t \in[0,1], s \in(0,1],
\end{array}
$$


which follow from (2.3) and (3.3) were utilized.

Similarly as in the proof of [16, Theorem III.4.1] we can complete the proof of the following assertion giving the relationship between the functions $U$ and $V$.

3.1. Proposition. Let us assume 2.1 and (3.1) and let the functions $U$ and $V$ be respectively given by (2.3) and (3.3). Then

$$
\begin{array}{rlrl}
V(t, s) & {\left[\mathrm{I}-\Delta^{+} \widetilde{A}(s)\right]\left[\mathrm{I}+\Delta^{+} A(s)\right]} & \\
& =\left[\mathrm{I}+\Delta^{-} \widetilde{A}(t)\right]\left[\mathrm{I}-\Delta^{-} A(t)\right] U(t, s) & & \text { if } 0 \leq s<t \leq 1, \\
V(t, t) & =U(t, t)=\mathrm{I} & & \text { for all } t \in[0,1], \\
V(t, s) & {\left[\mathrm{I}+\Delta^{-} \widetilde{A}(s)\right]\left[\mathrm{I}-\Delta^{-} A(s)\right]} & \\
& =\left[\mathrm{I}-\Delta^{+} \widetilde{A}(t)\right]\left[\mathrm{I}+\Delta^{+} A(t)\right] U(t, s) & & \text { if } 0 \leq t<s \leq 1 .
\end{array}
$$

3.2. Corollary. Under the assumptions of Proposition 3.1 the following relations are true

$$
\begin{aligned}
& V(0,0)=U(0,0)=\mathrm{I}, \\
& V(t, 0)\left[\mathrm{I}+\Delta^{+} A(0)\right] \quad=\left[\mathrm{I}-\left(\Delta^{-} A(t)\right)^{2}\right] U(t, 0) \text { for } t \in(0,1) \text {, } \\
& V(1,0)\left[\mathrm{I}+\Delta^{+} A(0)\right] \quad=\left[\mathrm{I}-\Delta^{-} A(1)\right] \quad U(1,0), \\
& V(1, s)\left[\mathrm{I}-\left(\Delta^{+} A(s)\right)^{2}\right]=\left[\mathrm{I}-\Delta^{-} A(1)\right] \quad U(1, s) \text { for } s \in(0,1) \text {, } \\
& V(1,1)=U(1,1)=\mathrm{I} \text {. }
\end{aligned}
$$

3.3. Proposition. Let us assume 2.1 and (3.1) and let the $m \times n$-matrix valued function $Z$ be given by

$$
\begin{aligned}
Z(s)= & K(1) \Delta^{-} A(1)\left[\mathrm{I}-\Delta^{-} A(1)\right]^{-1} V(1, s) \\
& +K(s)+\int_{s}^{1} K(\tau) \mathrm{d}_{\tau}[V(\tau, s)], \quad s \in[0,1] .
\end{aligned}
$$

Then a couple $(y, \delta) \in \mathbb{B V}^{n} \times \mathbb{R}^{m}$ is a solution to the problem (2.14)-(2.16) if and only if

$$
y^{T}(s)=-\delta^{T} Z(s) \quad \text { on }[0,1]
$$

and

$$
\delta^{T}\left(M+\int_{0}^{1} K(\tau) \mathrm{d}_{\tau} U(\tau, 0)\right)=0 .
$$

Proof. Since $\left(\left[\mathrm{I}-\Delta^{-} A(1)\right]^{-1}-\mathrm{I}\right)\left[\mathrm{I}-\Delta^{-} A(1)\right]=\Delta^{-} A(1)$, we have

$$
\left[\mathrm{I}-\Delta^{-} A(1)\right]^{-1}-\mathrm{I}=\Delta^{-} A(1)\left[\mathrm{I}-\Delta^{-} A(1)\right]^{-1} \text {. }
$$


A couple $(y, \delta) \in \mathbb{B V}^{n} \times \mathbb{R}^{m}$ is a solution to (2.14)-(2.16) if and only if $y$ is given on $[0,1]$ by (3.2), where $\eta \in \mathbb{R}^{n}$ is such that (2.15) and (2.16) are satisfied. Inserting (3.2) into (2.16) we obtain

$$
\eta^{\mathrm{T}}=-\delta^{\mathrm{T}} K(1)\left[\mathrm{I}-\Delta^{-} A(1)\right]^{-1} .
$$

Thus, making use of (3.2) and of the relation (3.11) we get

$$
\begin{aligned}
y^{\mathrm{T}}(s)= & -\delta^{\mathrm{T}} K(1)\left(\left[\mathrm{I}-\Delta^{-} A(1)\right]^{-1}-\mathrm{I}\right) V(1, s) \\
& -\delta^{\mathrm{T}}\left(K(s)+\int_{s}^{1} K(\tau) \mathrm{d}_{\tau}[V(\tau, s)]\right)=-\delta^{\mathrm{T}} Z(s) \text { for all } s \in[0,1] .
\end{aligned}
$$

Consequently, (3.2) reduces to (3.8). In particular, we have

$$
\begin{aligned}
y^{\mathrm{T}}(0)= & -\delta^{\mathrm{T}} K(1) \Delta^{-} A(1)\left[\mathrm{I}-\Delta^{-} A(1)\right]^{-1} V(1,0) \\
& -\delta^{\mathrm{T}}\left(K(0)+\int_{0}^{1} K(\tau) \mathrm{d}_{\tau}[V(\tau, 0)]\right) .
\end{aligned}
$$

Taking into account (3.6) this yields

$$
\begin{aligned}
y^{\mathrm{T}}(0)\left[\mathrm{I}+\Delta^{+} A(0)\right]=-\delta^{\mathrm{T}} K(1) \Delta^{-} A(1) U(1,0) \\
\\
\quad-\delta^{\mathrm{T}}\left(K(0)+\int_{0}^{1} K(\tau) \mathrm{d}_{\tau}[V(\tau, 0)]\right)\left[\mathrm{I}+\Delta^{+} A(0)\right] .
\end{aligned}
$$

Moreover, by (3.6) we also have

$$
\begin{aligned}
\int_{0}^{1} K(\tau) \mathrm{d}_{\tau}[V(\tau, 0)]\left[\mathrm{I}+\Delta^{+} A(0)\right] \\
\quad=\int_{0}^{1} K(\tau) \mathrm{d}_{\tau}[U(\tau, 0)]-K(1) \Delta^{-} A(1) U(1,0)-K(0) \Delta^{+} A(0) .
\end{aligned}
$$

Inserting (3.12) into (3.11) and (2.15) we finally obtain

$$
y^{\mathrm{T}}(0)\left[\mathrm{I}+\Delta^{+} A(0)\right]+\delta^{\mathrm{T}}[K(0)-M]=-\delta^{\mathrm{T}}\left(M+\int_{0}^{1} K(\tau) \mathrm{d}_{\tau}[U(\tau, 0)]\right),
$$

wherefrom the proof of the proposition immediately follows.

3.4. Corollary. Let us assume 2.1 and (3.1). Then

$$
\operatorname{dim} \mathcal{N}(\stackrel{*}{\mathrm{~L}})=m-\operatorname{rank}\left(M+\int_{0}^{1} K(\tau) \mathrm{d}_{\tau}[U(\tau, 0)]\right) .
$$


Proof. Denote

$$
r^{*}=m-\operatorname{rank}\left(M+\int_{0}^{1} K(\tau) \mathrm{d}_{\tau}[U(\tau, 0)]\right) .
$$

Then the system (3.9) possesses exactly $r^{*}$ linearly independent solutions. It is easy to see that if $\left\{\delta^{[1]}, \delta^{[2]}, \ldots, \delta^{\left[r^{*}\right]}\right\}$ is an arbitrary basis of the space of solutions to $(3.9)$, then the set of couples

$$
\left\{\left(-Z^{\mathrm{T}}(s) \delta^{[1]}, \delta^{[1]}\right),\left(-Z^{\mathrm{T}}(s) \delta^{[2]}, \delta^{[2]}\right), \ldots,\left(-Z^{\mathrm{T}}(s) \delta^{\left[r^{*}\right]}, \delta^{\left[r^{*}\right]}\right)\right\}
$$

with $Z$ given by $(3.7)$ is a basis in $\mathcal{N}\left(\mathrm{L}^{*}\right)$.

3.5. Corollary. Let us assume 2.1 and (3.1). Then

$$
\operatorname{dim} \mathcal{N}(\stackrel{*}{\mathrm{~L}})=\operatorname{dim} \mathcal{N}(\mathrm{L})+m-n
$$

Proof. Obviously, $x \in \mathcal{N}(\mathrm{L})$ if and only if

$$
x(t)=U(t, 0) c \quad \text { on }[0,1] \quad \text { and } \quad\left(M+\int_{0}^{1} K(\tau) \mathrm{d}_{\tau}[U(\tau, 0)]\right) c=0 .
$$

This implies that

$$
\operatorname{dim} \mathcal{N}(\mathrm{L})=n-\operatorname{rank}\left(M+\int_{0}^{1} K(\tau) \mathrm{d}_{\tau}[U(\tau, 0)]\right)
$$

The proof of our assertion then follows by Corollary 3.4.

3.6. Lemma. Let us assume 2.1 and (3.1). Let $Z \in \mathbb{B V}^{m \times n}$ be given by (3.7) and let

$$
\operatorname{rank}([K(0)-M, K(1)])=m,
$$

where $[K(1), K(0)-M]$ stands for the $m \times 2 n$-matrix formed in a usual way. Then

$$
\delta^{T} Z(s) \equiv 0 \quad \text { on } \quad[0,1]
$$

if and only if $\delta=0 \in \mathbb{R}^{m}$. 
Proof. Let $\delta \in \mathbb{R}^{m}$ be such that (3.15) holds. In particular, we have

$$
0=\delta^{\mathrm{T}} Z(1)=\delta^{\mathrm{T}} K(1)\left(\mathrm{I}+\Delta^{-} A(1)\left[\mathrm{I}-\Delta^{-} A(1)\right]^{-1}\right) .
$$

Moreover, since $\left(\mathrm{I}+\Delta^{-} A(1)\left[\mathrm{I}-\Delta^{-} A(1)\right]^{-1}\right)\left[\mathrm{I}-\Delta^{-} A(1)\right]=\mathrm{I}$, we have also

$$
\mathrm{I}+\Delta^{-} A(1)\left[\mathrm{I}-\Delta^{-} A(1)\right]^{-1}=\left[\mathrm{I}-\Delta^{-} A(1)\right]^{-1},
$$

and $\delta^{\mathrm{T}} Z(1)=\delta^{\mathrm{T}} K(1)\left[\mathrm{I}-\Delta^{-} A(1)\right]^{-1}=0$. This is possible only if

$$
\delta^{\mathrm{T}} K(1)=0 .
$$

On the other hand, inserting (3.7) and $s=0$ into (3.15) and making use of (3.9), (3.12) and (3.17), we obtain

$$
\begin{aligned}
0 & =\delta^{\mathrm{T}}\left(K(0)+\int_{0}^{1} K(\tau) \mathrm{d}_{\tau}[V(\tau, 0)]\right)\left[\mathrm{I}+\Delta^{+} A(0)\right] \\
& =\delta^{\mathrm{T}}\left(K(0)+\int_{0}^{1} K(\tau) \mathrm{d}_{\tau}[U(\tau, 0)]\right)=\delta^{\mathrm{T}}(K(0)-M),
\end{aligned}
$$

wherefrom, according to (3.17), the relation

$$
\delta^{\mathrm{T}}[K(0)-M, K(1)]=0
$$

follows. By the assumption (3.14) this is possible only if $\delta=0$.

3.7. Remark. Obviously, in the case of periodic conditions (2.18) (i.e. $m=n$, $M=0$ and $K(t) \equiv \mathrm{I}$ on $[0,1])$ the assumption (3.14) of Lemma 3.6 is satisfied. The relationship between linearly independent solutions of the linear algebraic system (3.9) and solutions of the adjoint boundary value problem (2.14)-(2.16) indicated in Lemma 3.6 could be extended to the general case, as well. Indeed, making use of (3.17) we obtain from (3.15) that $z(t)=K^{\mathrm{T}}(s) \delta$ has to satisfy the Volterra-Stieltjes integral equation

$$
z^{\mathrm{T}}(s)+\int_{0}^{1} z^{\mathrm{T}}(\tau) \mathrm{d}_{\tau}[V(\tau, s)]=0 \text { on }[0,1] .
$$

Since under our assumptions we have

$$
\operatorname{det}(\mathrm{I}+(V(s, s+)-V(s, s)))=\operatorname{det}\left(\mathrm{I}-\Delta^{+} A(s)\right) \neq 0 \text { for } s \in(0,1]
$$

and

$$
\operatorname{det}(\mathrm{I}+(V(0,0+)-V(0,0)))=\operatorname{det}(\mathrm{I})=1,
$$

it could be shown analogously as it was done in similar situations in the proofs of [16, Theorem II.3.10] or of [20, Theorem 5.5] that (3.18) may be true only if $z(s) \equiv 0$ on $[0,1]$. 


\section{Periodic problem}

In this section we will consider the periodic problem (0.1), (2.18) as well as the corresponding homogeneous problem (4.1), (2.18), where

$$
x(t)-x(0)-\int_{0}^{t} \mathrm{~d}[A(s)] x(s)=0, \quad t \in[0,1] .
$$

Obviously, the following assertion is true.

4.1. Proposition. Let $A \in \mathbb{B V}_{r e g}^{n \times n}, f \in \mathbb{G}_{r e g}^{n}$ and

$$
\operatorname{det}\left(\mathrm{I}-\Delta^{-} A(t)\right) \neq 0 \text { for all } t \in(0,1] \text {. }
$$

Then a function $x \in \mathbb{G}_{\text {reg }}^{n}$ is a solution to (0.1), (2.18) if and only if there is $c \in \mathbb{R}^{n}$ such that $x(t)$ is given on $[0,1]$ by (2.2) and

$$
[U(1,0)-\mathrm{I}] c=b,
$$

where

$$
b=\int_{0}^{1} \mathrm{~d}_{\tau}[U(1, \tau)](f(\tau)-f(0))-(f(1)-f(0)) .
$$

Furthermore, from Proposition 3.3, Corollary 3.4 and Lemma 3.6 the next assertion follows.

4.2. Proposition. Let us assume 2.1 and (3.1). Then both the homogeneous problem (4.1), (2.18) and its adjoint (2.19), (2.20) have exactly

$$
n-\operatorname{rank}[\mathrm{I}-U(1,0)]
$$

linearly independent solutions. A function $y \in \mathbb{B V}^{n}$ is a solution to the adjoint problem if and only if there is a $\delta \in \mathbb{R}^{n}$ such that

$$
y^{T}(s)=-\delta^{T}\left[\mathrm{I}-\Delta^{-} A(1)\right]^{-1} V(1, s) \text { on }[0,1]
$$

where $V$ is given by (3.3) and $\delta$ verifies the system

$$
\delta^{T}[U(1,0)-\mathrm{I}]=0 .
$$

Proof. It remains to show that in the case of periodic boundary conditions (i.e. $M=0$ and $K(t) \equiv \mathrm{I}$ on $[0,1])$ the formula $(3.7)$ reduces to

$$
Z(s)=\left[\mathrm{I}-\Delta^{-} A(1)\right]^{-1} V(1, s) \text { for } s \in[0,1] .
$$


Indeed, inserting $K(t) \equiv \mathrm{I}$ on $[0,1]$ into (3.7) and taking into account (3.16) we obtain

$$
\begin{aligned}
Z(s) & =\Delta^{-} A(1)\left[\mathrm{I}-\Delta^{-} A(1)\right]^{-1} V(1, s)+\mathrm{I}+V(1, s)-V(s, s) \\
& =\left(\mathrm{I}+\Delta^{-} A(1)\left[\mathrm{I}-\Delta^{-} A(1)\right]^{-1}\right) V(1, s) \\
& =\left[\mathrm{I}-\Delta^{-} A(1)\right]^{-1} V(1, s) \text { for all } s \in[0,1] . \square
\end{aligned}
$$

4.3. Definition. For a given function $A \in \mathbb{B V}^{n \times n}$, the symbol $A_{\mathrm{c}}$ stands for the continuous part of the function $A$. Furthermore, by $\Phi$ we denote the fundamental matrix solution corresponding to the equation

$$
x(t)-x(0)-\int_{0}^{t} \mathrm{~d}\left[A_{\mathrm{c}}(s)\right] x(s)=0, \quad t \in[0,1],
$$

i.e. $\Phi$ is the $n \times n$-matrix valued function defined by the relation

$$
\Phi(t)=\mathrm{I}+\int_{0}^{t} \mathrm{~d}\left[A_{\mathrm{c}}(\tau)\right] \Phi(\tau) \text { for } t, s \in[0,1] .
$$

Finally, by $\mathrm{S}(A)$ we denote the set of points of discontinuity of $A$ in $[0,1]$, i.e.

$$
\mathrm{S}(A)=\left\{t \in[0,1] ; \Delta^{-} A(t) \neq 0 \text { or } \Delta^{+} A(t) \neq 0\right\} .
$$

4.4. Remark. Obviously, $\Phi \in \mathbb{B V}^{n \times n} \cap \mathbb{C}^{n \times n}$. Furthermore, if $A_{\mathrm{c}}$ is absolutely continuous on [0,1], i.e. if there is an $n \times n$-matrix valued function $B(t)$ Lebesgue integrable on $[0,1]$ and such that

$$
A_{\mathrm{c}}(t)=\int_{0}^{t} B(\tau) \mathrm{d} \tau \text { on }[0,1]
$$

then $\Phi$ is the fundamental matrix solution of the ordinary differential equation

$$
x^{\prime}-B(t) x=0
$$

such that $\Phi(0)=\mathrm{I}$. In the general case $A_{\mathrm{c}} \in \mathbb{B V}^{n \times n} \cap \mathbb{C}^{n \times n}$, a sequence $\left\{A_{k}(t)\right\}_{k=1}^{\infty}$ of piecewise linear functions may be constructed (cf. [12]) in such a way that $\Phi$ is on $[0,1]$ the uniform limit of the sequence of fundamental solutions corresponding to ordinary differential equations

$$
x^{\prime}-A_{k}^{\prime} x=0
$$

In addition to assumptions 2.1 and (3.1) we will need the following assumptions, as well. 
4.5. Assumptions. $\mathrm{S}(A) \cup\{0\} \cup\{1\}=\left\{\tau_{k}\right\}_{k=0}^{p}$, where $p \in \mathbb{N}$ and $0=\tau_{0}<$ $\tau_{1}<\cdots<\tau_{p}=1$.

It is easy to see that if $A \in \mathbb{B V}_{\text {reg }}^{n \times n}$ fulfils (4.2) and 4.5 then there exist $n \times n$-matrices $C_{k}, k=0,1, \ldots, p$, such that

$$
A_{\mathrm{b}}(t):=A(t)-A_{\mathrm{c}}(t)=2 C_{0}\left(h(t)-\frac{1}{2}\right)+\sum_{k=1}^{p} 2 C_{k} h\left(t-\tau_{k}\right) \text { on }[0,1]
$$

where

$$
h(\tau)= \begin{cases}0 & \text { if } t<0 \\ \frac{1}{2} & \text { if } t=0 \\ 1 & \text { if } t>0\end{cases}
$$

Since obviously $\Delta^{+} A\left(\tau_{k}\right)=\Delta^{-} A\left(\tau_{k}\right)=C_{k}$ for $k=0,1, \ldots, p$, it is

$$
\operatorname{det}\left(\mathrm{I}-C_{k}\right) \neq 0 \text { for } k=1, \ldots, p \text {. }
$$

Furthermore, we have

$$
\begin{aligned}
U(t, s)=\left[\mathrm{I}-\Delta^{-}\right. & A(t)]^{-1} \Phi(t) \Pi_{\ell}^{k} \Phi^{-1}(s)\left[\mathrm{I}+\Delta^{+} A(s)\right] \\
& \text { if } t \in\left(\tau_{k-1}, \tau_{k}\right] \text { and } s \in\left[\tau_{\ell-1}, \tau_{\ell}\right) \\
& \text { for some } k, \ell \in\{1,2, \ldots, p\} \text { such that } k \geq \ell,
\end{aligned}
$$

where

$$
\Pi_{\ell}^{k}=\left\{\begin{array}{lr}
\mathrm{I} & \text { if } k \leq \ell, \\
\prod_{j=1}^{k-\ell}\left(\Phi^{-1}\left(\tau_{k-j}\right)\left[\mathrm{I}+C_{k-j}\right]\left[\mathrm{I}-C_{k-j}\right]^{-1} \Phi\left(\tau_{k-j}\right)\right) & \text { if } k>\ell .
\end{array}\right.
$$

(A similar formula was derived for the case of $A$ right-continuous on $(0,1]$ by Z. Wyderka, cf. [21].)

In particular, we have

$$
U(1,0)-\mathrm{I}=\left[\mathrm{I}-C_{p}\right]^{-1} \Phi(1) \Pi_{1}^{p}\left[\mathrm{I}+C_{0}\right]-\mathrm{I} .
$$

This enables us to complete the proofs of the following assertions providing conditions for the existence of solution to the homogeneous problem (4.1), (2.18)in terms of $\Phi$ and $C_{k}$ which are analogous to the results obtained by Z. Wyderka in [21] for the case of $A$ right continuous on $(0,1]$.

4.6. Proposition. Let us assume 2.1, (3.1) and 4.5 and let

$$
\Pi_{\ell}^{p}, \quad \ell=1,2, \ldots, p+1
$$


be given by (4.7). Then there exists the inverse matrix $\left(\Pi_{1}^{p}\right)^{-1}$ to $\Pi_{1}^{p}$ and is given by

$$
\left(\Pi_{1}^{p}\right)^{-1}=\prod_{j=1}^{p-1}\left(\Phi^{-1}\left(\tau_{j}\right)\left[\mathrm{I}-C_{j}\right]\left[\mathrm{I}+C_{j}\right]^{-1} \Phi\left(\tau_{j}\right)\right) .
$$

Moreover, the homogeneous problem (4.1), (2.18) has a non-trivial solution if and only if

$$
\operatorname{det}\left(\Phi(1)-\left[\mathrm{I}-C_{p}\right]\left[\mathrm{I}+C_{0}\right]^{-1}\left(\Pi_{1}^{p}\right)^{-1}\right)=0
$$

holds.

Proof. It was mentioned above that under our assumptions all the matrices $\left[\mathrm{I}-C_{k}\right], k=1, \ldots, p$, are invertible. Of course, assumptions 2.1 and (3.1) ensure the existence of the inverse matrices $\left[\mathrm{I}+C_{k}\right]^{-1}$ for $k=0,1, \ldots, p-1$, as well. Hence the matrix (4.9) is well defined and $\Pi_{1}^{p}\left(\Pi_{1}^{p}\right)^{-1}=\left(\Pi_{1}^{p}\right)^{-1} \Pi_{1}^{p}=\mathrm{I}$. The relation (4.8) may be modified as follows:

$$
\begin{aligned}
& U(1,0)-\mathrm{I}= \\
& {\left[\mathrm{I}-C_{p}\right]^{-1}\left(\Phi(1)-\left[\mathrm{I}-C_{p}\right]\left[\mathrm{I}+C_{0}\right]^{-1}\left(\Pi_{1}^{p}\right)^{-1}\right) \Pi_{1}^{p}\left[\mathrm{I}+C_{0}\right]}
\end{aligned}
$$

and it is easy to see that $\operatorname{det}(U(1,0)-\mathrm{I})=0$ holds if and only if the condition (4.10) is satisfied.

4.7. Proposition. Let the assumptions of Proposition 4.6 be satisfied and, moreover, let

$$
\Phi(1)=\left[\mathrm{I}-C_{p}\right]\left[\mathrm{I}+C_{0}\right]^{-1}\left(\Pi_{1}^{p}\right)^{-1} .
$$

Then any solution $x$ of the homogeneous equation (4.1) on $[0,1]$ is a solution to the problem (4.1), (2.18), as well.

Proof. Inserting (4.12) into (4.11), we get $U(1,0)=\mathrm{I}$.

For the nonhomogeneous problem $(0.1),(2.18)$ we have the following assertions.

4.8. Proposition. Let the assumptions of Proposition 4.6 hold. Then the problem $(0.1),(2.18)$ possesses a solution if and only if

$$
\eta^{T} \Phi(1)\left(\sum_{\ell=1}^{p}\left(\Pi_{\ell}^{p} \int_{\tau_{\ell-1}}^{\tau_{\ell}} \Phi^{-1}(\tau) \mathrm{d}[f(\tau)]\right)\right)=0
$$

holds for all $\eta \in \mathbb{R}^{n}$ such that

$$
\eta^{T}\left(\Phi(1)-\left[\mathrm{I}-C_{p}\right]\left[\mathrm{I}+C_{0}\right]^{-1}\left(\Pi_{1}^{p}\right)^{-1}\right)=0 .
$$


Proof. By Corollary 2.7, Proposition 4.2 and relation (4.11) the problem (0.1), (2.18) possesses a solution if and only if

$$
\eta^{\mathrm{T}} \int_{0}^{1} V(1, \tau) \mathrm{d} f(\tau)=0
$$

holds for any $\eta \in \mathbb{R}^{n}$ fulfilling (4.14). By (3.6) we have

$$
\begin{aligned}
V(1,0) & =\left[\mathrm{I}-C_{p}\right] U(1,0)\left[\mathrm{I}+C_{0}\right]^{-1} \\
V(1, \tau) & =\left[\mathrm{I}-C_{p}\right] U(1, \tau)\left[\mathrm{I}+\Delta^{+} A(\tau)\right]^{-1}\left[\mathrm{I}-\Delta^{-} A(\tau)\right]^{-1} \text { if } 0<\tau<1 \\
V(1,1) & =\mathrm{I}
\end{aligned}
$$

Inserting (4.6) into (4.16) we obtain

$$
\begin{aligned}
& V(1, \tau)=\Phi(1) \Pi_{\ell}^{p} \Phi^{-1}(\tau)\left[\mathrm{I}-\Delta^{-} A(\tau)\right]^{-1} \\
& \qquad \text { for } \ell=1,2, \ldots, p \text { and } \tau \in\left[\tau_{\ell-1}, \tau_{\ell}\right) .
\end{aligned}
$$

Now, define

$$
W_{\ell}(\tau)=\Pi_{\ell}^{p} \Phi^{-1}(\tau) \text { for } \tau \in\left[\tau_{\ell-1}, \tau_{\ell}\right] \text { and } \ell=1,2, \ldots, p \text {. }
$$

Making use of the relations

$$
\left(\left[\mathrm{I}-C_{\ell}\right]^{-1}-\mathrm{I}\right)=C_{\ell}\left[\mathrm{I}-C_{\ell}\right]^{-1}, \quad \ell=1,2, \ldots, p-1,
$$

and

$$
\Pi_{\ell}^{p}=\Pi_{\ell+1}^{p} \Phi^{-1}\left(\tau_{\ell}\right)\left[\mathrm{I}+C_{\ell}\right]\left[\mathrm{I}-C_{\ell}\right]^{-1} \Phi\left(\tau_{\ell}\right), \quad \ell=1,2, \ldots, p .
$$

we obtain for $\ell \in\{1,2, \ldots, p\}$

$$
V(1, \tau)-\Phi(1) W_{\ell}(\tau)=0 \quad \text { if } \quad \tau \in\left(\tau_{\ell-1}, \tau_{\ell}\right)
$$

Furthermore,

$$
\begin{aligned}
& V\left(1, \tau_{\ell-1}\right)-\Phi(1) W_{\ell}\left(\tau_{\ell-1}\right) \\
& \quad=\Phi(1) \Pi_{\ell}^{p} \Phi^{-1}\left(\tau_{\ell-1}\right)\left(\left[\mathrm{I}-C_{\ell-1}\right]^{-1}-\mathrm{I}\right) \\
& \quad=\Phi(1) \Pi_{\ell}^{p} \Phi^{-1}\left(\tau_{\ell-1}\right) C_{\ell-1}\left[\mathrm{I}-C_{\ell-1}\right]^{-1} \text { if } \ell>1
\end{aligned}
$$

and

$$
\begin{aligned}
& V\left(1, \tau_{\ell}\right)-\Phi(1) W_{\ell}\left(\tau_{\ell}\right) \\
& \quad=\Phi(1)\left(\Pi_{\ell+1}^{p} \Phi^{-1}\left(\tau_{\ell}\right)\left[\mathrm{I}-C_{\ell}\right]^{-1}-\Pi_{\ell}^{p} \Phi^{-1}\left(\tau_{\ell}\right)\right) \\
& \quad=\Phi(1) \Pi_{\ell+1}^{p} \Phi^{-1}\left(\tau_{\ell}\right)\left(\left[\mathrm{I}-C_{\ell}\right]^{-1}-\left[\mathrm{I}+C_{\ell}\right]\left[\mathrm{I}-C_{\ell}\right]^{-1}\right) \\
& \quad=-\Phi(1) \Pi_{\ell+1}^{p} \Phi^{-1}\left(\tau_{\ell}\right) C_{\ell}\left[\mathrm{I}-C_{\ell}\right]^{-1} \quad \text { if } \quad \ell<p .
\end{aligned}
$$

Moreover, we have 


$$
V(1,0)-\Phi(0) W_{1}(0)=V(1,1)-\Phi(1) W_{p}(1)=0 .
$$

Thus,

$$
\begin{gathered}
\int_{0}^{\tau_{1}}\left(V(1, \tau)-\Phi(1) W_{1}(\tau)\right) \mathrm{d}[f(\tau)]=-\Phi(1) \Pi_{2}^{p} \Phi^{-1}\left(\tau_{1}\right) C_{1}\left[\mathrm{I}-C_{1}\right]^{-1} \\
\int_{\tau_{\ell-1}}^{\tau_{\ell}}\left(V(1, \tau)-\Phi(1) W_{\ell}(\tau)\right) \mathrm{d}[f(\tau)] \\
=\Phi(1)\left(\Pi_{\ell}^{p} \Phi^{-1}\left(\tau_{\ell-1}\right) C_{\ell-1}\left[\mathrm{I}-C_{\ell-1}\right]^{-1}-\Pi_{\ell+1}^{p} \Phi^{-1}\left(\tau_{\ell}\right) C_{\ell}\left[\mathrm{I}-C_{\ell}\right]^{-1}\right) \\
\quad \text { for } \ell=2,3, \ldots, p-1
\end{gathered}
$$

and

$$
\begin{aligned}
\int_{\tau_{p-1}}^{1} & \left(V(1, \tau)-\Phi(1) W_{p}(\tau)\right) \mathrm{d}[f(\tau)] \\
= & \Phi(1) \Phi^{-1}\left(\tau_{p-1}\right) C_{p-1}\left[\mathrm{I}-C_{p-1}\right]^{-1}
\end{aligned}
$$

Consequently,

$$
\begin{aligned}
& \int_{0}^{1} V(1, \tau) \mathrm{d}[f(\tau)]-\sum_{\ell=1}^{p} \Phi(1)\left(\int_{\tau_{\ell-1}}^{\tau_{\ell}} W_{\ell}(\tau) \mathrm{d}[f(\tau)]\right) \\
& =\sum_{\ell=1}^{p}\left(\int_{\tau_{\ell-1}}^{\tau_{\ell}}\left(V(1, \tau)-\Phi(1) W_{\ell}(\tau)\right) \mathrm{d}[f(\tau)]\right) \\
& =\Phi(1)\left(\sum_{\ell=2}^{p}\left(\Pi_{\ell}^{p} \Phi^{-1}\left(\tau_{\ell-1}\right) C_{\ell-1}\left[\mathrm{I}-C_{\ell-1}\right]^{-1} \Delta^{+} f\left(\tau_{\ell-1}\right)\right)\right) \\
& \quad-\Phi(1)\left(\sum_{\ell=1}^{p-1}\left(\Pi_{\ell+1}^{p} \Phi^{-1}\left(\tau_{\ell}\right) C_{\ell}\left[\mathrm{I}-C_{\ell}\right]^{-1} \Delta^{-} f\left(\tau_{\ell}\right)\right)\right)=0,
\end{aligned}
$$

wherefrom the proof of our proposition immediately follows.

4.9. Corollary. Let the assumptions of Proposition 4.6 be satisfied. Then the problem (0.1), (2.18) possesses a unique solution for any $f \in \mathbb{G}_{\text {reg }}^{n}$ if and only if

$$
\operatorname{det}\left(\Phi(1)-\left[\mathrm{I}-C_{p}\right]\left[\mathrm{I}+C_{0}\right]^{-1}\left(\Pi_{1}^{p}\right)^{-1}\right) \neq 0
$$

is satsified with $\left(\Pi_{1}^{p}\right)^{-1}$ is given by (4.9).

4.10. Corollary. Let the assumptions of Proposition 4.6 be satisfied. Then the problem $(0.1),(2.18)$ possesses a solution if and only if

$$
\eta^{T}\left[\mathrm{I}-C_{p}\right]\left[\mathrm{I}+C_{0}\right]^{-1}\left(\sum_{\ell=1}^{p}\left(\left(\Pi_{1}^{\ell}\right)^{-1} \int_{\tau_{\ell-1}}^{\tau_{\ell}} \Phi^{-1}(\tau) \mathrm{d}[f(\tau)]\right)\right)=0
$$

holds for all $\eta \in \mathbb{R}^{n}$ verifying (4.14). 
Proof. Since by (4.7) and (4.9)

$$
\left(\Pi_{1}^{p}\right)^{-1} \Pi_{\ell}^{p}=\prod_{j=1}^{\ell-1}\left(\Phi^{-1}\left(\tau_{j}\right)\left[\mathrm{I}-C_{j}\right]\left[\mathrm{I}+C_{j}\right]^{-1} \Phi\left(\tau_{j}\right)\right)=\left(\Pi_{1}^{\ell}\right)^{-1},
$$

the proof follows by inserting of

$$
\eta^{\mathrm{T}} \Phi(1)=\eta^{\mathrm{T}}\left[\mathrm{I}-C_{p}\right]\left[\mathrm{I}+C_{0}\right]^{-1}\left(\Pi_{1}^{p}\right)^{-1}
$$

into (4.13).

4.11. Remark. By Proposition 4.1 the problem (0.1), (2.18) has a solution if and only if

$$
\delta^{\mathrm{T}}\left(\int_{0}^{1} \mathrm{~d}_{\tau}[U(1, \tau)](f(\tau)-f(0))-(f(1)-f(0))\right)=0
$$

holds for any $\delta \in \mathbb{R}^{n}$ fulfilling (4.4). According to (3.6) we have

$$
U(1, \tau)=\left[\mathrm{I}-C_{p}\right]^{-1} V(1, \tau)[\mathrm{I}+Q(\tau)] \text { on }[0,1]
$$

where

$$
Q(\tau)=\left\{\begin{array}{cl}
\Delta^{+} A(0) & \text { if } \tau=0 \\
-\left(\Delta^{+} A(\tau)\right)^{2}, & \text { if } \tau \in(0,1) \\
-\Delta^{-} A(1) & \text { if } \tau=1
\end{array}\right.
$$

Since obviously $Q(0+)=Q(\tau+)=Q(\tau-)=Q(1-)=0$ for all $\tau \in(0,1)$, it is

$$
\begin{aligned}
\int_{0}^{1} \mathrm{~d}_{\tau}[V(1, \tau) Q(\tau)](f(\tau)-f(0)) & =V(1,1) Q(1)(f(1)-f(0)) \\
& =-V(1,1) \Delta^{-} A(1)(f(1)-f(0)) .
\end{aligned}
$$

Thus, making use of (3.16) we obtain

$$
\begin{aligned}
\int_{0}^{1} \mathrm{~d}_{\tau}[U(1, \tau)](f(\tau)-f(0))-(f(1)-f(0)) \\
=\left[\mathrm{I}-C_{p}\right]^{-1} \int_{0}^{1} \mathrm{~d}_{\tau}[V(1, \tau)](f(\tau)-f(0)) \\
\quad \quad\left(\mathrm{I}+C_{p}\left[\mathrm{I}-C_{p}\right]^{-1}\right)(f(1)-f(0) \\
=\left[\mathrm{I}-C_{p}\right]^{-1}\left(\int_{0}^{1} \mathrm{~d}_{\tau}[V(1, \tau)](f(\tau)-f(0))-(f(1)-f(0))\right) .
\end{aligned}
$$


Finally, integrating-by-parts and taking into account that according to (3.4) we have $V(1,.) \in \mathbb{B V}_{\text {reg }}^{n \times n}, V(1,0+)=V(1,0)$ and $V(1,1-)=V(1,1)$, we get that

$$
\begin{gathered}
\delta^{\mathrm{T}}\left(\int_{0}^{1} \mathrm{~d}_{\tau}[U(1, \tau)](f(\tau)-f(0))-(f(1)-f(0))\right) \\
=\delta^{\mathrm{T}}\left[\mathrm{I}-C_{p}\right]^{-1} \int_{0}^{1} V(1, \tau) \mathrm{d}[f(\tau)]
\end{gathered}
$$

is true for any $\delta \in \mathbb{R}^{n}$ and any $f \in \mathbb{G}_{\text {reg }}^{n}$. It follows immediately that the condition (4.17) is satisfied for any $\delta \in \mathbb{R}^{n}$ such that (4.4) is true if and only if (4.15) holds for any $\eta \in \mathbb{R}^{n}$ such that (4.14) is true. By the proof of Proposition 4.8 it means that the condition (4.17) is satisfied for any $\delta \in \mathbb{R}^{n}$ verifying (4.4) is true if and only if (4.13) is true for any $\eta \in \mathbb{R}^{n}$ satisfying (4.14).

4.12. Remark. If

$$
\left(\Delta^{+} A(t)\right)^{2}=\left(\Delta^{-} A(t)\right)^{2}=0 \text { for all } t \in(0,1)
$$

then

$$
\left[\mathrm{I}-\Delta^{-} A(t)\right]\left[\mathrm{I}+\Delta^{+} A(t)\right]=\mathrm{I}-\left(\Delta^{+} A(t)\right)^{2}=\mathrm{I} \text { for all } t \in(0,1) .
$$

In particular, for any $j=1,2, \ldots, p-1$ we have

$$
\begin{array}{ll}
{\left[\mathrm{I}-C_{j}\right]^{-1}=\left[\mathrm{I}+C_{j}\right],} & {\left[\mathrm{I}+C_{j}\right]^{-1}=\left[\mathrm{I}-C_{j}\right],} \\
C_{j}\left[\mathrm{I}-C_{j}\right]^{-1}=C_{j}, & C_{j}\left[\mathrm{I}+C_{j}\right]=C_{j}, \\
{\left[\mathrm{I}-C_{j}\right]^{2}=\mathrm{I}-2 C_{j},} & {\left[\mathrm{I}+C_{j}\right]^{2}=\mathrm{I}+2 C_{j} .}
\end{array}
$$

This enables us to simplify the necessary and sufficient condition for the existence of a solution to the periodic problem (0.1), (2.18) given in Proposition 4.8.

4.13. Corollary. Let the assumptions of Proposition 4.6 be satisfied and let (4.18) hold. Then the problem (0.1), (2.18) possesses a solution if and only if

$$
\eta^{T} \Phi(1)\left(\sum_{\ell=1}^{p} \Pi_{\ell}^{p} \int_{\tau_{\ell-1}}^{\tau_{\ell}} \Phi^{-1}(\tau) \mathrm{d}[f(\tau)]\right)=0
$$

holds for all $\eta \in \mathbb{R}^{n}$ satisfying the system (4.14), where

$$
\left(\Pi_{1}^{p}\right)^{-1}=\prod_{j=1}^{p-1}\left(\Phi^{-1}\left(\tau_{j}\right)\left[\mathrm{I}-2 C_{j}\right] \Phi\left(\tau_{j}\right)\right)
$$

and

$$
\Pi_{1}^{p}=\prod_{j=1}^{p-1}\left(\Phi^{-1}\left(\tau_{p-j}\right)\left[\mathrm{I}+2 C_{p-j}\right] \Phi\left(\tau_{p-j}\right)\right) .
$$


4.14. Remark. The results obtained in this paper may be obviously adapted to the case of an arbitrary subinterval $\left[t_{0}, T\right]$ in the place of $[0,1]$. Furthermore, let the functions $A:\left[t_{0}, \infty\right) \mapsto \mathbb{R}^{n \times n}$ and $f:\left[t_{0}, \infty\right) \mapsto \mathbb{R}^{n}$ be locally of bounded variation, while the set $\mathrm{S}(A)$ of the points of discontinuity of $A$,

$$
\mathrm{S}(A)=\left\{\tau_{j}\right\}_{j \in \mathbb{M}}, \quad \text { where either } \mathbb{M}=\mathbb{N} \quad \text { or } \quad \mathbb{M}=\left\{1,2, \ldots, \nu_{A}\right\} \varsubsetneqq \mathbb{N}
$$

is ordered in such a way that $t_{0}<\tau_{1}<\cdots<\tau_{j-1}<\tau_{j}<\tau_{j+1} \cdots<\infty$ holds for any $j \in \mathbb{M}$ such that $j+1 \in \mathbb{M}$ and the unique accumulation point of $\mathrm{S}(A)$ may be $\infty$. Furthermore, let us assume that

$$
\operatorname{det}\left(\mathrm{I}-\Delta^{-} A(t)\right) \neq 0 \text { for all } t \in\left[t_{0}, \infty\right)
$$

and $A$ generates an $\omega$-periodic measure i.e. $\omega>0$ and $A(t+\omega)-A(t)=$ const. on $\left[t_{0}, \infty\right)$. Notice that by $[21$, Lemma 1.4] the function $A$ generates an $\omega$-periodic measure if and only if there is a constant matrix $B_{0} \in \mathbb{R}^{n \times n}$ and an $\omega$-periodic function $B$ locally of bounded variation on $\left[t_{0}, \infty\right)$ and such that

$$
A(t)=\frac{1}{\omega} B_{0} t+B(t) \text { on }\left[t_{0}, \infty\right)
$$

and there is $k_{0} \in \mathbb{N}$ such that

$$
\tau_{j+k_{0}}=\tau_{j}, \Delta^{-} A\left(\tau_{j+k_{0}}\right)=\Delta^{-} A\left(\tau_{j}\right) \text { and } \Delta^{+} A\left(\tau_{j+k_{0}}\right)=\Delta^{+} A\left(\tau_{j}\right)
$$

for all $j \in \mathbb{N}$.

It is easy to see that if $A$ and $f$ generate $\omega$-periodic measures and if they are regular on $\left(t_{0}, t_{0}+\omega\right)$ and $A$ is continuous at $t_{0}$, then the problem to find an $\omega$-periodic solution to (0.1) is equivalent to the boundary value problem (4.1), (2.18) (with the interval $\left[t_{0}, t_{0}+\omega\right]$ in place of $\left.[0,1]\right)$ fulfilling the assumptions of this section.

4.15. Example. Consider the second order differential equation with distributional coefficients

$$
u^{\prime \prime}-\left(\alpha t+2 q h\left(t-\tau_{1}\right)\right)^{\prime} u=g^{\prime},
$$

where $0<\tau_{1}<1, \alpha=a^{2}, a>0, h$ stands for the Heaviside type function given by (4.5), $g \in \mathbb{G}_{\mathrm{reg}}$ and $q \in \mathbb{R}$ is a parameter. The corresponding periodic problem (4.19),

$$
u(0)=u(1), \quad u^{\prime}(0)=u^{\prime}(1)
$$


may be rewritten in the form $(4.1),(2.18)$, where

$$
\begin{gathered}
A(t)=A_{0} t+2 C_{1} h\left(t-\tau_{1}\right) \\
A_{0}=\left(\begin{array}{cc}
0 & 1 \\
a^{2} & 0
\end{array}\right), C_{1}=\left(\begin{array}{ll}
0 & 0 \\
q & 0
\end{array}\right) \text { and } f(t)=\left(\begin{array}{c}
0 \\
g(t)
\end{array}\right) .
\end{gathered}
$$

In particular, we have $n=p=2, \mathrm{~S}(A)=\left\{\tau_{1}\right\}$, where $0=\tau_{0}<\tau_{1}<\tau_{2}=1$. Moreover, it is

$$
C_{1}^{2}=0, \operatorname{det}\left(\mathrm{I}-C_{1}\right)=1 \text { and }\left(\Pi_{1}^{p}\right)^{-1}=\left(\Pi_{1}^{2}\right)^{-1}=\Phi\left(\tau_{1}\right)\left(\mathrm{I}-2 C_{1}\right) \Phi^{-1}\left(\tau_{1}\right),
$$

where the fundamental matrix $\Phi$ corresponding to $A_{\mathrm{c}}(t)=A_{0} t$ is for any $a>0$ given on $[0,1]$ by

$$
\Phi(t)=\left(\begin{array}{cc}
\frac{\mathrm{e}^{a t}+\mathrm{e}^{-a t}}{2} & \frac{\mathrm{e}^{a t}-\mathrm{e}^{-a t}}{2 a} \\
\frac{a\left(\mathrm{e}^{a t}-\mathrm{e}^{-a t}\right)}{2} & \frac{\mathrm{e}^{a t}+\mathrm{e}^{-a t}}{2}
\end{array}\right) .
$$

Inserting (4.21) into $\left(\Pi_{1}^{2}\right)^{-1}$, we get

$$
\left(\Pi_{1}^{2}\right)^{-1}=\left(\begin{array}{cc}
1+\frac{q\left(\mathrm{e}^{2 a \tau_{1}}-\mathrm{e}^{-2 a \tau_{1}}\right)}{2 a} & \frac{q\left(\mathrm{e}^{a \tau_{1}}-\mathrm{e}^{-a \tau_{1}}\right)^{2}}{2 a^{2}} \\
-\frac{q\left(\mathrm{e}^{a \tau_{1}}+\mathrm{e}^{-a \tau_{1}}\right)^{2}}{2} & 1-\frac{q\left(\mathrm{e}^{2 a \tau_{1}}-\mathrm{e}^{-2 a \tau_{1}}\right)}{2 a}
\end{array}\right) .
$$

Denote

$$
M(q):=\Phi(1)-\left(\Pi_{1}^{2}\right)^{-1}
$$

It is easy to verify that

and

$$
\operatorname{det}(M(q))=2-\mathrm{e}^{a}-\mathrm{e}^{-a}-\frac{q\left(\mathrm{e}^{a}-\mathrm{e}^{-a}\right)}{a}
$$

$$
\operatorname{det}(M(q))=0 \quad \text { if and only if } \quad q=q^{*}:=a \frac{2-\mathrm{e}^{a}-\mathrm{e}^{-a}}{\mathrm{e}^{a}-\mathrm{e}^{-a}}
$$

By Corollary 4.9 the problem (4.19), (4.20) possesses a unique solution for any $g \in \mathbb{G}_{r e g}$ if and only if $q \neq q^{*}$. Furthermore,we have

$$
M\left(q^{*}\right)=\frac{\mathrm{e}^{\frac{a}{2}}-\mathrm{e}^{-\frac{a}{2}}}{2\left(\mathrm{e}^{\frac{a}{2}}+\mathrm{e}^{-\frac{a}{2}}\right)} M_{0}^{*}
$$


where

$$
M_{0}^{*}=\left(\begin{array}{cc}
\left(\mathrm{e}^{a}-\mathrm{e}^{-a}\right)+\left(\mathrm{e}^{2 a \tau_{1}}-\mathrm{e}^{-2 a \tau_{1}}\right) & \frac{\left(\mathrm{e}^{\frac{a}{2}}+\mathrm{e}^{-\frac{a}{2}}\right)^{2}+\left(\mathrm{e}^{a \tau_{1}}+\mathrm{e}^{-a \tau_{1}}\right)^{2}}{a} \\
a\left(\left(\mathrm{e}^{\frac{a}{2}}+\mathrm{e}^{-\frac{a}{2}}\right)^{2}-\left(\mathrm{e}^{a \tau_{1}}+\mathrm{e}^{-a \tau_{1}}\right)^{2}\right) & \left(\mathrm{e}^{a}-\mathrm{e}^{-a}\right)-\left(\mathrm{e}^{2 a \tau_{1}}-\mathrm{e}^{-2 a \tau_{1}}\right)
\end{array}\right)
$$

and it is easy to see that $\eta^{\mathrm{T}} M\left(q^{*}\right)=0$ holds for $\eta \in \mathbb{R}^{2}$ if and only if there is $\gamma \in \mathbb{R}$ such that

$$
\eta^{\mathrm{T}}=\gamma\left(-a \frac{\mathrm{e}^{a}-\mathrm{e}^{2 a \tau_{1}}}{\mathrm{e}^{a}+\mathrm{e}^{2 a \tau_{1}}}, 1\right) .
$$

Consequently, Corollary 4.13 yields that in the case $q=q^{*}$ the problem (4.19), (4.20) possesses a solution if and only if the relation

$$
\left(-a \frac{\mathrm{e}^{a}-\mathrm{e}^{2 a \tau_{1}}}{\mathrm{e}^{a}+\mathrm{e}^{2 a \tau_{1}}}, 1\right) \int_{0}^{1} \Phi^{-1}(s) \mathrm{d}[f(s)]=0
$$

is satisfied. It is easy to check that under our assumptions this condition reduces to

$$
\int_{0}^{\tau_{1}}\left(\mathrm{e}^{a+a s}+\mathrm{e}^{2 a \tau_{1}-a s}\right) \mathrm{d}[g(s)]+\int_{\tau_{1}}^{1}\left(\mathrm{e}^{a s}+\mathrm{e}^{2 a \tau_{1}+a-a s}\right) \mathrm{d}[g(s)]=0 .
$$

4.16. Example. Consider the problem from Example 4.15, but with $\alpha=-a^{2}$ and $a>0$. In this case we have is given by

$$
\Phi(t)=\left(\begin{array}{cc}
\cos (a t) & \frac{\sin (a t)}{a} \\
-a \sin (a t) & \cos (a t)
\end{array}\right), \quad t \in[0,1],
$$

and

$$
\begin{aligned}
M(q) & =\Phi(1)-\left(\Pi_{1}^{2}\right)^{-1} \\
& =\left(\begin{array}{cc}
-1+\cos (a)-\frac{q \sin \left(2 a \tau_{1}\right)}{a} & \frac{\sin (a)}{a}+\frac{q\left(\cos \left(2 a \tau_{1}\right)-1\right)}{a^{2}} \\
-a \sin (a)+q\left(\cos \left(2 a \tau_{1}\right)+1\right) & -1+\cos (a)+\frac{q\left(\sin \left(2 a \tau_{1}\right)\right)}{a}
\end{array}\right) .
\end{aligned}
$$

It can be verified that

$$
\operatorname{det}(M(q))=2\left(1-\cos (a)-\frac{q \sin (a)}{a}\right)
$$

and thus $\operatorname{det}(M(q))=0$ if and only if either there exists $k \in \mathbb{N}$ such that $a=2 k \pi$ or $a \neq k \pi$ for all $k \in \mathbb{N}$ and $q=q^{*}:=a \tan \left(\frac{a}{2}\right)$. 
By Corollary 4.9 it follows again that the given problem has a unique solution for any $g \in \mathbb{G}_{\text {reg }}$ if and only if there is a $k \in \mathbb{N}$ such that $a=(2 k+1) \pi$ or $a \neq k \pi$ for all $k \in \mathbb{N}$ and $q \neq q^{*}$.

If $a \neq k \pi$ for all $k \in \mathbb{N}$ and $q=q^{*}$, then

$$
M\left(q^{*}\right)=2 \tan \left(\frac{a}{2}\right) M_{0}^{*},
$$

where

$$
M_{0}^{*}=\left(\begin{array}{cc}
-\sin \left(\frac{a}{2}+a \tau_{1}\right) \cos \left(\frac{a}{2}-a \tau_{1}\right) & \frac{\cos \left(\frac{a}{2}+a \tau_{1}\right) \cos \left(\frac{a}{2}-a \tau_{1}\right)}{a} \\
a \sin \left(\frac{a}{2}+a \tau_{1}\right) \sin \left(\frac{a}{2}-a \tau_{1}\right) & -\cos \left(\frac{a}{2}+a \tau_{1}\right) \sin \left(\frac{a}{2}-a \tau_{1}\right)
\end{array}\right) .
$$

Moreover, $\eta^{\mathrm{T}} M\left(q^{*}\right)=0$ for $\eta \in \mathbb{R}^{2}$ if and only if there is a $\gamma \in \mathbb{R}$ such that $\eta^{\mathrm{T}}=\gamma \zeta^{\mathrm{T}}$, where

$$
\zeta^{\mathrm{T}}=\left\{\begin{array}{llll}
\left(a \tan \left(\frac{a}{2}-a \tau_{1}\right), 1\right) & \text { if } \tau_{1} \neq \frac{1}{2}-\frac{\pi(2 \ell+1)}{2 a} & \text { for all } & \ell \in \mathbb{Z}, \\
\left(1, \frac{\cot \left(\frac{a}{2}-a \tau_{1}\right)}{a}\right) & \text { if } \tau_{1} \neq \frac{1}{2}-\frac{\pi \ell}{a} & \text { for all } & \ell \in \mathbb{Z}, \\
(1,0) & \text { if } \tau_{1}=\frac{1}{2}-\frac{\pi(2 \ell+1)}{2 a} & \text { for some } & \ell \in \mathbb{Z}, \\
(0,1) & \text { if } \tau_{1}=\frac{1}{2}-\frac{\pi \ell}{a} & \text { for some } & \ell \in \mathbb{Z} .
\end{array}\right.
$$

and $\mathbb{Z}$ stands as usual for the set of integers.

It can be shown that in the first case (i.e. $\tau_{1} \neq \frac{1}{2}-\frac{\pi(2 \ell+1)}{2 a}$ for all $\ell \in \mathbb{Z}$ ) the necessary and sufficient condition for the existence of a solution to the problem reduces to

$$
\int_{0}^{\tau_{1}} \cos \left(\frac{a}{2}-a\left(\tau_{1}-s\right)\right) \mathrm{d}[g(s)]+\int_{\tau_{1}}^{1} \cos \left(\frac{a}{2}+a\left(\tau_{1}-s\right)\right) \mathrm{d}[g(s)]=0 .
$$

Similar necessary and sufficient conditions for the existence of a solution could be derived in all the remaining cases.

Acknowledgement. The author wishes to thank to Professor Jan Ligesza for suggestions which lead to a substantial improvement of this paper.

\section{References}

[1] Aumann G.: Reelle Funktionen. Springer-Verlag, Berlin, 1969.

[2] Bainov D., Simeonov P.: Impulsive differential equations: periodic solutions and applications. Pitman Monographs and Surveys in Pure and Applied Mathematics 66, Longman Scientific, Harlow, 1993. 
[3] Boichuk A. A., Perestyuk N. A., Samoilenko A. M.: Periodic solutions of impulsive differential equations in critical cases (in Russian). Diff. Uravn. 27 (1991), 1516-1521.

[4] Fraňková D.: Regulated functions. Math. Bohem. 116 (1991), 20-59.

[5] Halperin I.: Introduction to the Theory of Distributions. University of Toronto Press, Toronto, 1952.

[6] Hildebrandt T. H.: Introduction to the Theory of Integration. Academic Press, New York-London, 1963.

[7] Hönig Ch.S.: Volterra Stieltjes-Integral Equations. North Holland and American Elsevier, Mathematics Studies 16, Amsterdam and New York, 1975.

[8] Kurzweil J.: Generalized ordinary differential equations and continuous dependence on a parameter. Czechoslovak Math. J. 7 (82) (1957), 418-449.

[9] Kurzweil J.: Nichtabsolute konvergente Integrale. B. G. Teubner Verlagsgesselschaft, Leipzig, 1980.

[10] Ligęza J.: Weak Solutions of Ordinary Differential Equations. Uniwersytet Sląski, Katowice, 1986.

[11] Pandit S. G., Deo S. G.: Differential Equations Involving Impulses. Lecture Notes in Mathematics 954, Springer-Verlag, Berlin, 1982.

[12] Pelant M.: On approximations of solutions of generalized differential equations (in Czech), Dissertation, Charles University. (Praha, 1997)

[13] Pelant M., Tvrdý M.: Linear distributional differential equations in the space of regulated functions. Math. Bohem. 118 (1993), 379-400.

[14] Rudin W.: Functional Analysis. McGraw-Hill, New York, 1973.

[15] Schwabik Š.: Generalized Ordinary Differential Equations. World Scientific, Singapore, 1992.

[16] Schwabik Š., Tvrdý M., Vejvoda O.: Differential and Integral Equations: Boundary Value Problems and Adjoints. Academia and D.Reidel, Praha and Dordrecht, 1979.

[17] Tvrdý M.: Regulated functions and the Perron-Stieltjes integral. Casopis Pěst. Mat. 114 (1989), 187-209.

[18] Tvrdý M.: Generalized differential equations in the space of regulated functions (Boundary value problems and controllability). Math. Bohem. 116 (1991), 225-244.

[19] Tvrdý M.: Linear bounded functionals on the space of regular regulated functions. Tatra Mountains Mathematical Publications 8 (1996), 203-210.

[20] Tvrdý M.: Linear integral equations in the space of regulated functions. Mathem. Bohem. 123 (1998), 177-212.

[21] Wyderka Z.: Periodic Solutions of Linear Differential Equations with Measures as Coefficients. Acta Univ. Palacki. Olomuc., Fac. re. nat. 35 (1996), 199-214. 